\title{
Preheating in New Inflation
}

\author{
Mariel Desroche, ${ }^{1}$ Gary N. Felder, ${ }^{1}$ Jan M. Kratochvil, ${ }^{2}$ and Andrei Linde ${ }^{2}$ \\ ${ }^{1}$ Department of Physics, Clark Science Center, \\ Smith College Northampton, MA 01063, USA \\ ${ }^{2}$ Department of Physics, Stanford University, Stanford, CA 94305-4060, USA
}

(Dated: October 25, 2018)

\begin{abstract}
During the last ten years a detailed investigation of preheating was performed for chaotic inflation and for hybrid inflation. However, nonperturbative effects during reheating in the new inflation scenario remained practically unexplored. We do a full analysis of preheating in new inflation, using a combination of analytical and numerical methods. We find that the decay of the homogeneous component of the inflaton field and the resulting process of spontaneous symmetry breaking in the simplest models of new inflation usually occurs almost instantly: for the new inflation on the GUT scale it takes only about 5 oscillations of the field distribution. The decay of the homogeneous inflaton field is so efficient because of a combined effect of tachyonic preheating and parametric resonance. At that stage, the homogeneous oscillating inflaton field decays into a collection of waves of the inflaton field, with a typical wavelength of the order of the inverse inflaton mass. This stage usually is followed by a long stage of decay of the inflaton field into other particles, which can be described by the perturbative approach to reheating after inflation. The resulting reheating temperature typically is rather low.
\end{abstract}

PACS numbers: $98.80 . \mathrm{Cq}$

SU-ITP-5/02

hep-ph/0501080 


\section{INTRODUCTION}

Inflationary cosmology solves a number of problems in the big bang model and is well supported by observational evidence. According to this theory the universe at the end of the inflationary period consisted almost entirely of the homogeneous inflaton field. After inflation this field decayed into inhomogeneous fluctuations and other forms of particles and fields, ultimately giving rise to the forms of matter that make up the universe today.

An understanding of this decay period, known as reheating, can provide crucial links between the inflationary epoch and the subsequent thermalized hot big bang era. The reheating period may have involved high energy phase transitions, symmetry breaking, baryogenesis, and other effects that could have observable signatures and that could give us insights into physics at energies beyond the reach of accelerator experiments.

Early discussions of reheating were based on the assumption that the homogeneous inflaton field decayed perturbatively as a collection of particles [1]. The perturbative mechanism typically requires thousands of oscillations of the inflaton field until it decays into usual elementary particles. More recently, however, it was discovered that coherent field effects such as parametric resonance can lead to the decay of the homogeneous field much faster than would have been predicted by perturbative methods, within few dozen oscillations [2]. These coherent effects produce high energy, nonthermal fluctuations that could have significance for understanding developments in the early universe, such as baryogenesis. This early stage of rapid nonperturbative decay may be followed by a period of slower, perturbative effects, so the rapid early stage is called preheating.

In 3] it was found that another effect known as tachyonic preheating can lead to even faster decay than parametric resonance. This effect occurs whenever the homogeneous field rolls down a tachyonic $\left(\left(d^{2} V / d \phi^{2}\right)<0\right)$ region of its potential. When that occurs a tachyonic, or spinodal instability leads to exponentially rapid growth of all long wavelength modes $\left(k^{2}<\left|\left(d^{2} V / d \phi^{2}\right)\right|\right)$. This growth can often drain all of the energy from the homogeneous field within a single oscillation.

We are now in a position to classify the dominant mechanisms by which the homogeneous inflaton field decays in different classes of inflationary models. The simplest of these models can be broken into three classes: small field, or new inflation models [4], large field, or 
chaotic inflation models [5], and multi-field, or hybrid models [6].

In simple chaotic inflation preheating is generally dominated by parametric resonance, although there are parameter ranges where this can not occur. ${ }^{1}$ Tachyonic preheating does not occur in these models because the curvature of the potential is always positive. In [3] it is shown that tachyonic preheating dominates the preheating phase in hybrid models of inflation. In this paper we explore preheating in new inflationary models. We find that for almost all realistic parameters tachyonic preheating works simultaneously with parametric resonance. The resulting effect is very strong, so that the homogeneous mode of the inflaton field typically decays within few oscillations.

We should emphasize, however, that this stage of reheating is not the last one. The inhomogeneities of the scalar field can be very long-living in the new inflation scenario; their decay can be described by the perturbative methods of Ref. [1].

We do not pretend that the taxonomy given above exhausts the space of all possible inflationary theories. One can imagine arbitrarily complicated models that combine aspects of small and large field models or theories with non-canonical kinetic terms, gravitational effects, and so on. Nonetheless, we believe that by understanding preheating in these three classes of theories we will have a good understanding of all of the simplest possibilities, and in many cases the more complex models will simply involve combinations of the effects seen in these simpler cases.

In Section II we give an analytic investigation of tachyonic preheating in the simplest model of new inflation. Section III describes parametric resonance which occurs in this model. A full treatment, which would unify these two effects and take into account other important effects which may occur at the later stages of preheating, requires lattice calculations [8], which we perform using the program LATTICEEASY [9]. In Section [V] we describe our lattice simulations and summarize their results, including the growth of fluctuations, the properties of the resulting spectra, and the formation of domain walls. Finally, we conclude with a summary of preheating in new inflation models and suggestions for further work.

\footnotetext{
${ }^{1}$ We refer to "simple" models as ones where the field simply oscillates around a minimum at $\phi=0$, such as $V=\frac{1}{2} m^{2} \phi^{2}$. In non-oscillatory models such as exponential potentials the situation is more complicated. See [] for a discussion of preheating in these models.
} 


\section{TACHYONIC PREHEATING IN NEW INFLATION}

In this paper we are considering tachyonic preheating in models of new inflation, i.e. models where the inflaton field rolls from a potential maximum at $\phi=0$ to a minimum at a symmetry breaking value $\phi=v$. For definiteness we focus primarily on the original new inflation model [4] based on the Coleman-Weinberg potential [10]

$$
V(\phi)=\frac{1}{4} \lambda \phi^{4}\left[\ln \frac{|\phi|}{v}-\frac{1}{4}\right]+\frac{1}{16} \lambda v^{4},
$$

but the results we present should apply to any new inflation model (with one small exception noted below.) The potential energy takes its maximum value $V_{0}=(1 / 16) \lambda v^{4}$ at $\phi=0$ and vanishes at the minima at $\phi= \pm v$. We are going to use units $M_{p}=1$, even though sometimes we will write $M_{p}$ explicitly.

During inflation the field is near the maximum. Roughly speaking, inflation ends when

$H^{2} \approx \frac{d^{2} V}{d \phi^{2}}$. Neglecting factors of order unity (including logarithmic factors) this occurs at a field value

$$
\phi_{0} \approx \frac{v^{2}}{M_{p}} .
$$

While this result is approximate, we have verified numerically that for a wide range of values of $v$ this field value corresponds to a time when kinetic energy is still orders of magnitude smaller than potential energy, so we can safely start our lattice simulations at this time without fear of missing important effects.

First we consider the homogeneous rolling field, neglecting backreaction. The energy lost to Hubble friction in one oscillation can be estimated as

$$
\Delta V \approx H \dot{\phi} \Delta \phi
$$

Up to factors of order unity we can approximate $H \approx \sqrt{V_{0}} / M_{p}, \dot{\phi} \approx \sqrt{V_{0}}$, and $\Delta \phi \approx v$, so

$$
\Delta V \approx V_{0} \frac{v}{M_{p}}
$$

Approximating the potential near the top as a negative quartic potential we can use this formula to find the field value $\phi_{1}$ after the first oscillation

$$
-\lambda \phi_{0}^{4}+\lambda \phi_{1}^{4}=V_{0} \frac{v}{M_{p}} \approx \lambda v^{4} \frac{v}{M_{p}}
$$




$$
\phi_{1} \approx v\left(\frac{v}{M_{p}}\right)^{1 / 4}
$$

Continued application of this argument leads to the conclusion that after $n$ oscillations the field value will be roughly

$$
\phi_{n} \approx v\left(\frac{n v}{M_{p}}\right)^{1 / 4}
$$

We wish now to estimate the growth of fluctuations due to tachyonic preheating. We consider preheating to be finished when $\delta \phi \approx v$, at which point it is meaningless to talk about oscillations of the homogeneous field. To estimate this growth we consider the growth of a single mode $\phi_{k}$, which to first order obeys the equation of motion

$$
\ddot{\phi}_{k}+\left(p^{2}+m_{e f f}^{2}\right) \phi_{k}=0
$$

where $m_{\text {eff }}^{2}=\left(d^{2} V / d \phi^{2}\right)$ is the effective mass of the field as a function of the value of the homogeneous field $\langle\phi\rangle$ and $p$ is the physical (not comoving) momentum. Because $m_{e f f}^{2}<0$ modes with $p^{2}<\left|m_{\text {eff }}^{2}\right|$ will experience exponential growth.

To estimate the speed of this growth we can use the following trick. If we consider a homogeneous field we can define a variable $D \equiv \dot{\phi}$ and write an equation of motion for $D$

$$
\begin{gathered}
\dot{D}+\frac{d V}{d \phi}=0 \\
\ddot{D}=-\frac{d^{2} V}{d \phi^{2}} \frac{d \phi}{d t}=-m_{e f f}^{2} D .
\end{gathered}
$$

Thus modes with $p^{2} \ll\left|m_{\text {eff }}^{2}\right|$ obey the same equation of motion as the derivative $\dot{\phi}$. We know the solution to the equation for $\dot{\phi}$ from energy conservation, though. Treating the potential as an inverted quartic and neglecting Hubble friction, $\dot{\phi} \propto \phi^{2}$. The growth of the fluctuations $\delta \phi$ is determined by the modes with $p^{2} \ll\left|m_{e f f}^{2}\right|$, so $\delta \phi$ also grows proportionally to $\phi^{2}$.

At the end of inflation

$$
\delta \phi \approx H \approx \frac{\sqrt{V_{0}}}{M_{p}} \approx \frac{\sqrt{\lambda} v^{2}}{M_{p}} .
$$

Thus after the field falls to the minimum at $\phi=v$

$$
\delta \phi \approx \frac{\sqrt{\lambda} v^{2}}{M_{p}}\left(\frac{v}{\phi_{0}}\right)^{2}=\sqrt{\lambda} M_{p} .
$$


Note that as the field rolls back up the potential the fluctuations will not lose this growth because the waves are decoherent.

This result says that for $v<\sqrt{\lambda}$, which is typically of order $10^{-6}$, preheating will complete in a single oscillation. For larger values of $v$ we must consider subsequent oscillations. In each one $\delta \phi$ grows by an amount $\left(v / \phi_{n}\right)^{2}$, which is roughly $\left(M_{p} / v\right)^{1 / 2}$, so after $n$ oscillations the fluctuations will have grown to roughly

$$
\delta \phi \approx \sqrt{\lambda} M_{p}\left(\frac{M_{p}}{v}\right)^{n / 2}
$$

The number of oscillations required for $\delta \phi$ to reach $v$ in this approximation is

$$
n \approx \frac{\ln \lambda}{\ln \frac{v}{M_{p}}} .
$$

For $\lambda=10^{-12}$, which corresponds to COBE normalization of density perturbations, and $v \sim 10^{-3} M_{p}$, which corresponds to the GUT scale, preheating will complete in roughly 5 oscillations. Of course when $\delta \phi \sim v$ the perturbative approximation will break down, but preheating will be over. As we will see, the numerical investigation confirms this simple estimate.

Qualitatively these results depend only on the fact that the inflaton feels an inverted polynomial potential as it rolls from its maximum at $\phi=0$ to the minimum at $\phi=v$. Thus the same type of behavior would result from an inverted quartic with or without logarithmic corrections, an inverted cubic potential, or nearly any other symmetry breaking potential. ${ }^{2}$

These estimates suggest that for any $v \ll 1$ tachyonic preheating will drain the energy from the homogeneous, oscillating, inflaton field within a few oscillations. We will see in the next section that there are important corrections to these rough estimates, and for $v$ sufficiently large tachyonic preheating does not operate, however for $v \lesssim 10^{-2}$ the behavior is qualitatively as described by these analytical estimates.

\footnotetext{
2 These results do not hold for the case of an inverted quadratic potential. However, if you assume that the potential near the maximum is an inverted quadratic then $\frac{d^{2} V}{d \phi^{2}}$ is constant as the field rolls, so inflation cannot end in this regime.
} 


\section{PARAMETRIC RESONANCE}

In addition to the tachyonic preheating, there is also a parametric resonance in this model. The most efficient part of this process occurs far from the minimum of the effective potential, where it was expected to happen in the earlier works on reheating in new inflation 11].

Indeed, let us find the range of the values of the field $\phi$, for which the adiabaticity condition is violated and a broad parametric resonance takes place.

The adiabaticity condition is $\dot{w}<w^{2}$. Here $w^{2}=k^{2}+V^{\prime \prime}=k^{2}+3 \lambda \phi^{2}\left(\ln \frac{|\phi|}{v}+\frac{1}{3}\right)$. Instead of investigating the general case, let us look at the vicinity of the point $\phi^{*}$, where $V^{\prime \prime}=0$, i.e. at the point where the low-momenta modes change their nature from normal to tachyonic. At that point $w^{2}=k^{2}$, whereas $\dot{w}=\frac{\lambda \phi \dot{\phi}}{k}$ and $\dot{\phi}^{2} \approx 0.05 \lambda v^{4}$. This gives a broad range of momenta $k$ for which the adiabaticity condition is violated:

$$
k \lesssim 0.5 \sqrt{\lambda} v=0.5 m
$$

where $m=\sqrt{\lambda} v$ is the mass of the inflaton field in the minimum of $V(\phi)$ at $\phi=v$. This means that the parametric resonance is powerful enough to excite all modes and create particles with momenta smaller than (the half of) the mass of the inflaton field at the minimum of its effective potential. These momenta, however, are much higher than the smallest momenta excited by tachyonic preheating.

But this is also the range of momenta where the tachyonic amplification of fluctuations is operative. In fact, when the scalar field moves in the region with $\phi<\phi^{*}$, its mass is tachyonic, so many of the modes produced due to strong nonadiabaticity near $\phi=\phi^{*}$ continue growing exponentially when the field moves in the region $\phi<\phi^{*}$.

As a result, parametric resonance and tachyonic growth amplify each other, which should lead to an even faster decay of the homogeneous scalar field, with the spectrum of produced fluctuations spanning a large range of momenta, from the inflationary Hubble constant $H \sim \sqrt{\lambda} v \frac{v}{M_{p}}$ to the mass of the field in the minimum of its effective potential $\sim \sqrt{\lambda} v$. The homogeneous scalar field $\phi$ should decay within just a few of its oscillations. In this sense, new inflation takes an intermediate position between hybrid inflation, where the decay is purely tachyonic and takes just a single oscillation, and the simplest versions of chaotic 
inflation scenario, where the decay is due to parametric resonance and occurs within few dozen oscillations. In what follows we will describe the results of numerical simulations of reheating in new inflation.

\section{NUMERICAL CALCULATIONS}

While the estimates in the previous section provide a useful general description of the process of tachyonic preheating in new inflation, a full analysis including backreaction and rescattering effects requires a lattice simulation. We performed a series of simulations of the Coleman-Weinberg model (11) using the LATTICEEASY program [9]. We used $\lambda=10^{-12}$, which is fixed by the normalization of the cosmic microwave background, but we varied $v$ as

a free parameter. In all of the figures in this section time is measured in units of $(\sqrt{\lambda} v)^{-1}$, wave number $k$ is measured in units of $\sqrt{\lambda} v$, and the inflaton field $\phi$ is measured in units of $v$. Note that $\sqrt{\lambda} v$ is the mass of the inflaton field in the minimum of the effective potential; we use the gravitational Planck mass $M_{p} \equiv 1 / \sqrt{G}$.

The number of gridpoints required to meet both of these requirements was quite large, which restricted us to doing one and two dimensional simulations. We verified for each case that the essential features were unchanged in going from one to two dimensions, so we tentatively infer that the same basic results would hold in three dimensions as well. In the appendix we give all of the parameters and other details about the simulations.

Figure 1 1 shows the evolution of the mean value of the inflaton field for $v=0.1 M_{p}$. The Hubble friction has a slightly greater effect on the field than our rough analytical estimates suggested. The result of this small correction is that after falling from the top of the potential the mean of the field never comes back up into the tachyonic region. Consequently tachyonic preheating and the parametric resonance described in the previous section does not occur for this high value of the symmetry breaking scale. Fluctuations of the field in this simulation never grew from their initial vacuum values. The decay of the homogeneous component of the inflaton field in this regime occurs very slowly. In the beginning, it may occur due to a very narrow and inefficient parametric resonance, as described in Section IV of Ref. 2], but then very rapidly this resonance completely disappears, and decay continues due to perturbative effects described in [1]. 


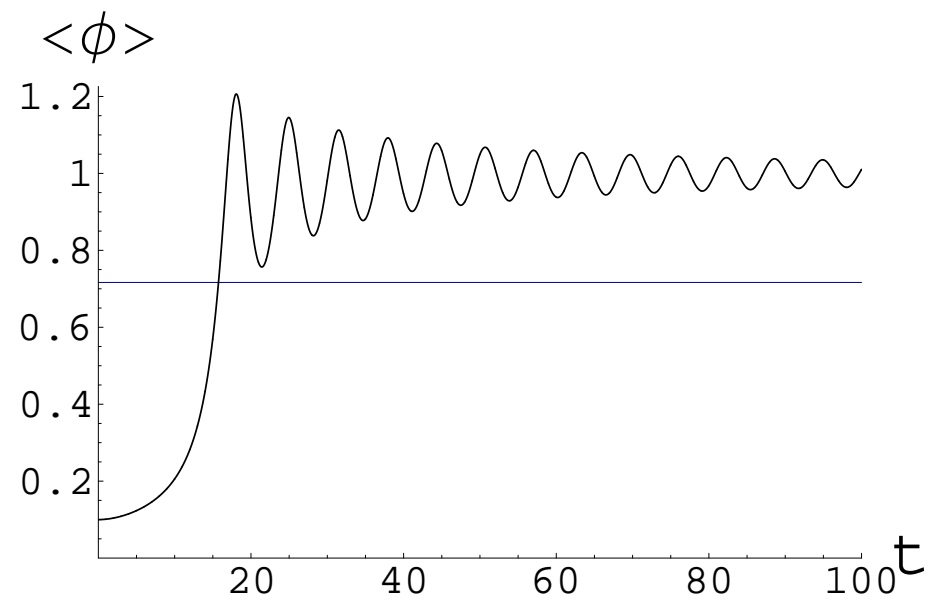

FIG. 1: Mean value of the inflaton field $\langle\phi\rangle$ (zero mode) for $v=10^{-1} M_{p}$. A horizontal line indicates the field value below which the potential is tachyonic (negative mass squared). The amplitude of the field here and in other figures is given in units of $v$, whereas the time $t$ is given in units $m^{-1}=(\sqrt{\lambda} v)^{-1}$, where $m$ is the mass of the scalar field near the minimum of the effective potential.

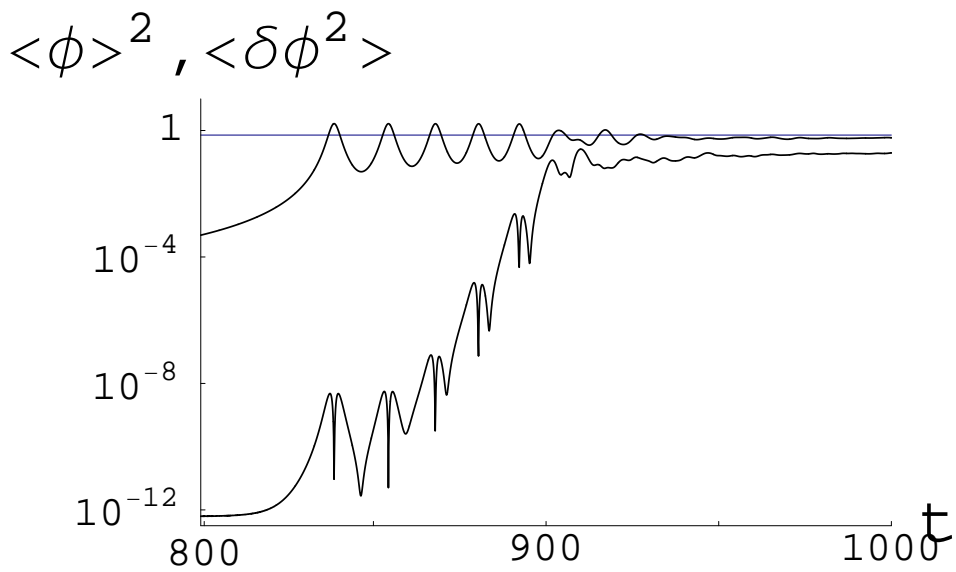

FIG. 2: Squared mean $\langle\phi\rangle^{2}$ and variance $\left\langle\delta \phi^{2}\right\rangle$ of the inflaton field for $v=10^{-3} M_{p}$. A horizontal line indicates the field value below which the potential is tachyonic (negative mass squared). As one can clearly see form this figure, the oscillations of the homogeneous component of the scalar field $\phi$ are completely damped out after the first 5 oscillations, whereas the variance remains much smaller than 1, in units of $v$. This means that the process of spontaneous symmetry breaking in this scenario completes within 5 oscillations.

For smaller values of the parameter $v$ the situation changes dramatically. Figure 2 shows 
the mean and variance of the inflaton field for $v=10^{-3} M_{p}$. In this plot you can clearly see that the mean field oscillates through the tachyonic region repeatedly, leading to rapid exponential growth of fluctuations. After about five or six such oscillations the zero mode is effectively destroyed. We did simulations of values of $v$ ranging from $10^{-1} M_{p}$ to $10^{-5} M_{p}$ and found that the cutoff below which tachyonic preheating was efficient was somewhere in between $10^{-2} M_{p}$ and $10^{-3} M_{p}$. Thus for nearly all realistic values of the parameters, tachyonic preheating may be expected to dominate the initial decay of the homogeneous inflaton field in models of the type we are considering. In the rest of this section we will focus on results for $v=10^{-3} M_{p}$ as an illustrative example, but we found these results to be typical for parameters for which tachyonic preheating was possible.
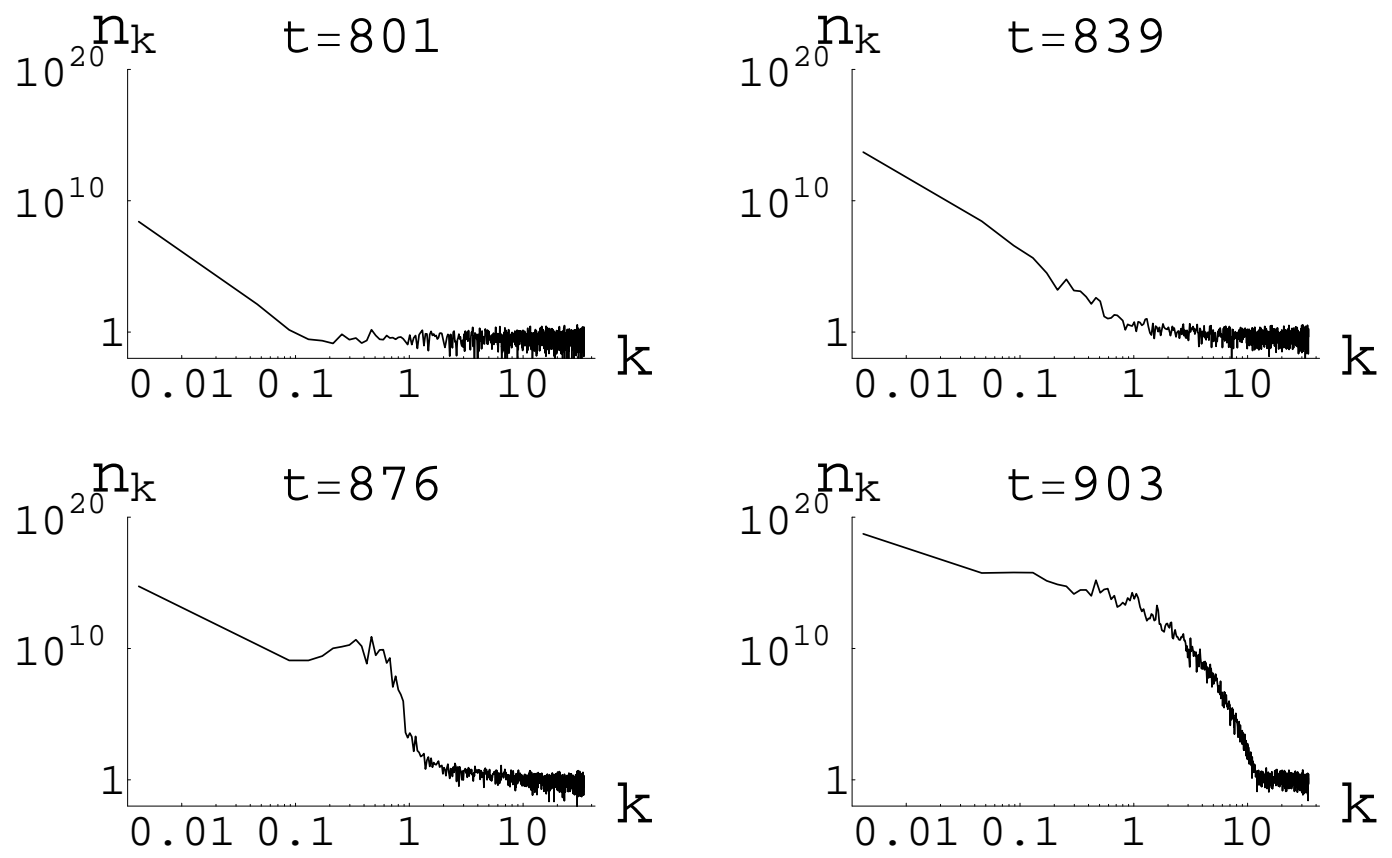

FIG. 3: Spectra of occupation number $n_{k}$ as a function of momentum $k$ at different times for $v=10^{-3} M_{p}$.

To explore the decay of the homogeneous inflaton in greater detail we can consider the occupation number $n_{k}$ of different modes. Figure 3 shows the development of this spectrum over time. At the first time shown the field has been slowly rolling near the top of the potential. Only very long wavelength modes have been excited by this point. In the next frame we see continued growth of long-wavelength modes due to tachyonic preheating. Tachyonic preheating can only amplify modes with $k<\left|m_{e f f}\right|$. The effective mass of the 
field varies from zero at the maximum to a minimum value of $\left|m_{e f f}\right| \approx 0.5 \sqrt{\lambda} v$, so modes with momenta above this cutoff were not excited. Even for modes below the cutoff, lower $k$ modes were excited more strongly than higher $k$ modes because the field spent more time in the regime where these low $k$ modes were tachyonic.

Following this initial stage we see the growth of higher $k$ modes, leading to the development of a peak around $k \approx \sqrt{\lambda} v$. The modes in this peak region are marginally able to be excited by tachyonic preheating. Moreover, tachyonic preheating would be expected to produce a monotonically decreasing spectrum like the one in the second frame of figure 3. rather than a peak such as we see in the third frame. This peak must therefore be the result of parametric resonance, as described in Section III. As a further check, we examined whether the formation of this peak was purely attributable to parametric resonance produced by the oscillating zero mode, or whether it was also influenced by scattering from the already-produced long-wavelength modes. To this end we solved the coupled equations for the evolution of the zero mode (neglecting backreaction) and a single mode from the peak.

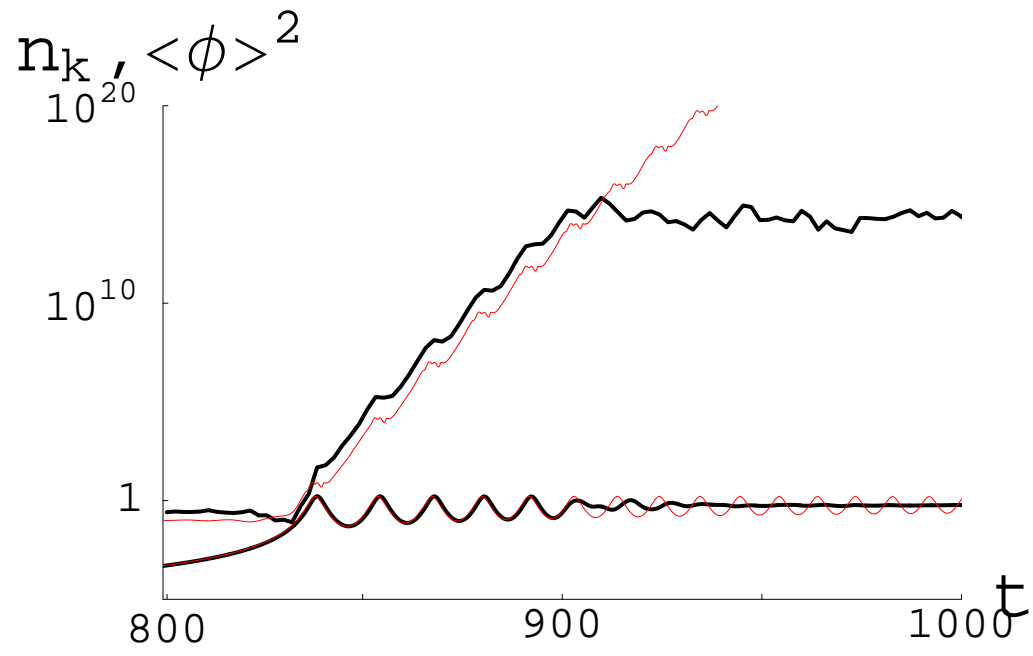

FIG. 4: Growth of a fluctuation in the peak $(k \approx 0.58)$. The lower curves show the evolution of the zero mode and the upper curves show the occupation number $n_{k}$ of this mode. The solid (black) curves show results from LATTICEEASY. The thin (red) curves show results from a Mathematica calculation in which the zero mode was evolved with no backreaction. These results confirm our expectations that the growth of the modes with $k \sim 0.5$ occur due to parametric resonance practically independently of the tachyon preheating.

Figure 4 compares the growth of this mode in the lattice simulation to its growth when 
coupled only to the zero mode. The two plots are nearly identical until the point when backreaction from the long-wavelength modes significantly affects the zero mode. Thus the formation of the peak in the third frame of figure 3 is purely a result of parametric resonance resulting from the oscillations of the zero mode. Note, however, that this plot also shows the need for lattice simulations; a linear analysis would not show the effects of backreaction and the point at which the decay of the homogeneous mode completes.

Figure 5 shows a zoomed in view of the growth of the mode depicted in figure 4. The growth of the occupation number occurs when the field is high on the potential hill and this growth alternates with periods of unchanging occupation number as the field moves through the minimum. This figure can be compared with the images showing broad parametric resonance in [2]. In both cases the growth occurs when $m_{\text {eff }}^{2}$ passes through zero, but in the case considered in [2] that occurred around the potential minimum, whereas here it occurs on the side of the potential hill.

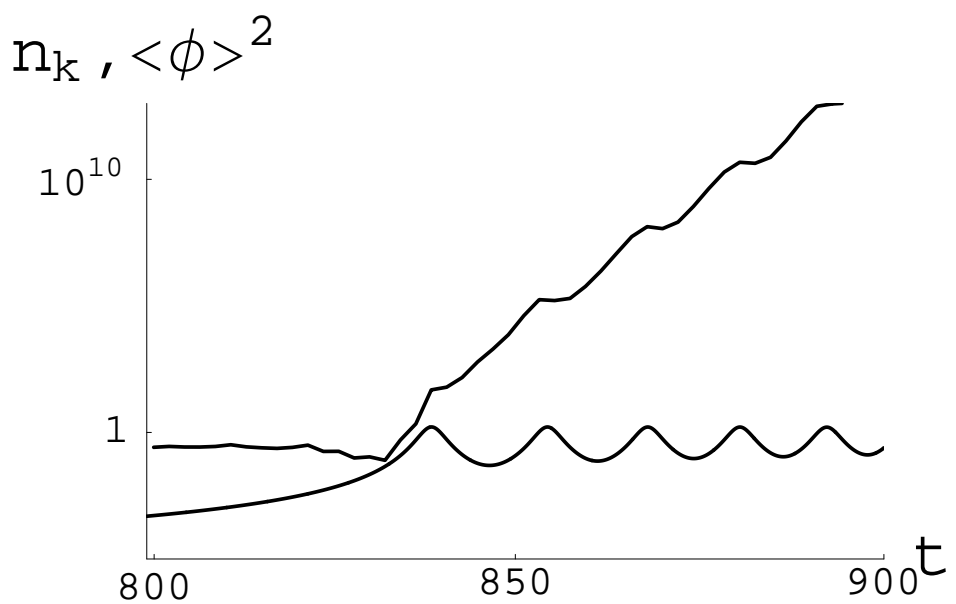

FIG. 5: This figure is the same as figure 4 except showing only the lattice results and zoomed in to show features more clearly. As we see, the exponential growth of the occupation numbers occurs each time when the average value of the scalar field becomes much smaller than $v$.

In summary, the fluctuations of the field pass through several distinct stages. Initially modes with $k \lesssim 0.5 \sqrt{\lambda} v$ are excited by tachyonic preheating. Later modes with $k \approx 0.5 \sqrt{\lambda} v$ are excited by parametric resonance with the still oscillating zero mode, producing a peak in the spectrum. Later still this peak smooths out due to rescattering, the oscillations of the zero mode cease, the peak in the spectrum is smoothed out, and we end up with a flat 
spectrum in the IR followed by an exponential cutoff near $k=v$, as shown in the final frame of figure 3. This is similar to the spectrum that would be produced by parametric resonance in a large-field inflationary model, but the decay is significantly more rapid in this case. As a result, the total energy available to the high energy modes is very large.

These processes are illustrated by a series of four panels in Fig. 6, which show a twodimensional distribution of the inflaton scalar field $\phi$ at different stages of our calculations. The simulation was done for $v=10^{-3} M_{p}$ on a $2048 \times 2048$ lattice, and the output was averaged over 8 lattice points for display in the figure.

The first of the four panels in Fig. 6 shows the distribution of the field $\phi$ at the time $t=740$, at the very early stages of rolling of the field towards the minimum of its effective potential. At that time the average amplitude of the field was still very small $\phi \approx 0.00755$. The interval of change shown in this panel is 0.0001. As we see, the amplitude of fluctuations of this field at that time is well below this level: The field looks almost completely homogeneous, apart for small quantum fluctuations which did not grow much at that stage.

The second panel shows the distribution of the field $\phi$ at the time $t=830$. The field still continues rolling down during the first oscillation, but the average amplitude of the field at that time has grown up to $\phi \approx 0.15395$. The interval of change shown in this panel is also 0.0001, as large as in the previous panel. We see that the long wavelength perturbations have already grown up significantly, and have magnitude $\delta \phi \sim 0.00005$.

The third panel shows the distribution of the field $\phi$ at the time $t=850$. At that time the field already made one oscillation and started its way down during the second oscillation; compare with Fig. 2. Its average amplitude at that time is $\phi \approx 0.39785$. The interval of change shown in this panel is 0.0002 , i.e. two times greater than in the previous panels. As we can see, the amplitude of the long wavelength perturbations experienced an additional growth, but new perturbations with a much shorter wavelength have grown up on top of the long wavelength perturbations.

Finally, the last panel in Fig. 6 shows the distribution of the field $t=1000$. At that time the short wavelength fluctuations completely dominate the field distribution. The average value of the scalar field $\langle\phi\rangle$ no longer oscillates. Its value is close to 1 , though it is slightly smaller than 1 because of the partial symmetry restoration due to the contribution of the fluctuations $\left\langle\phi^{2}\right\rangle$ to the effective potential. The energy of the field $\phi$ is concentrated in the 
waves of the field $\phi$ with momenta $k \lesssim 1$ produced by amplification of quantum fluctuations during preheating.
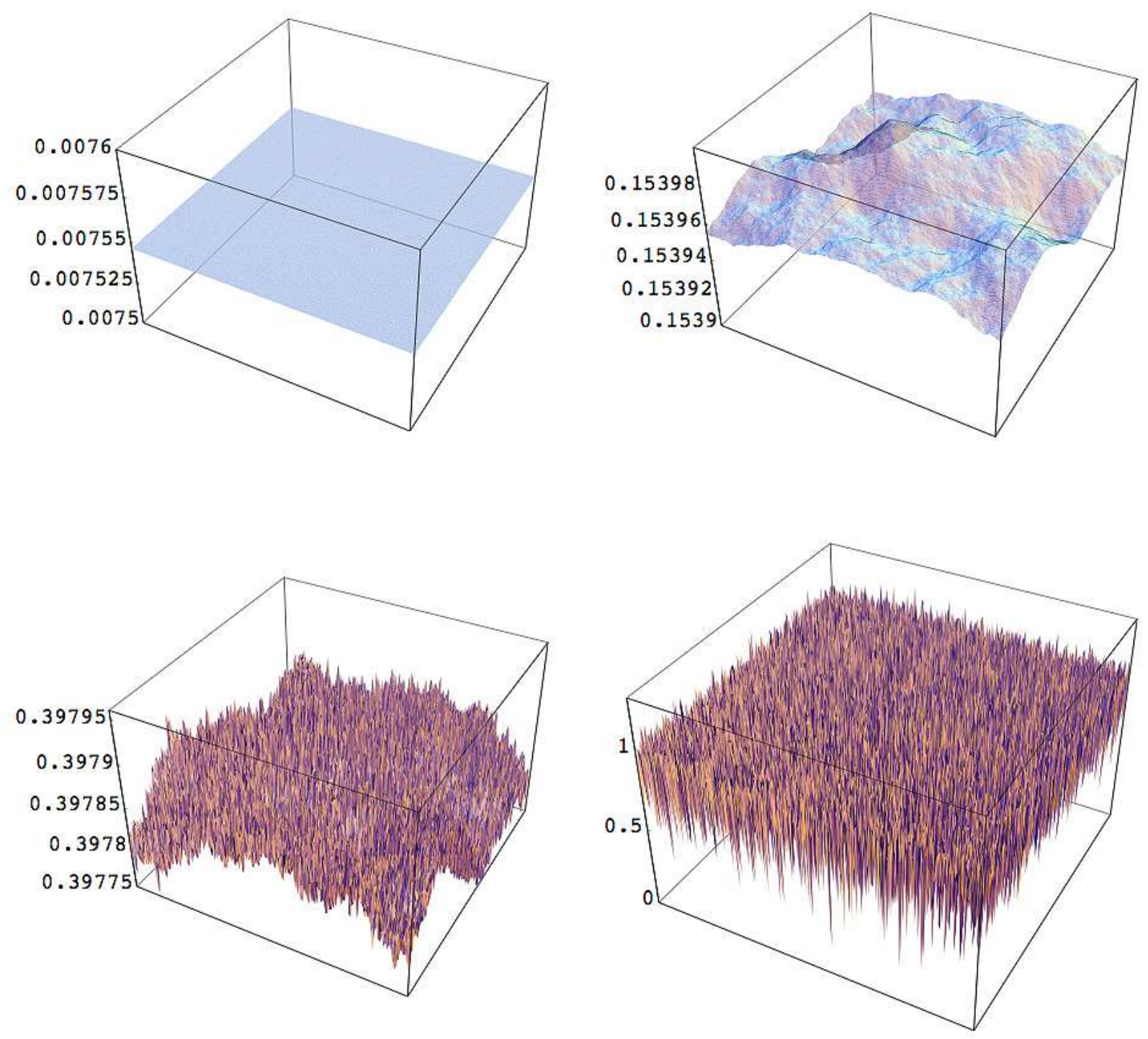

FIG. 6: Lattice images of a two-dimensional simulation with $v=10^{-3} M_{p}$. In the beginning of the process, the scalar field $\phi$ is practically homogeneous due to inflation, see the first panel. When it starts falling down, tachyonic instability generates long wavelength fluctuations of the scalar field, shown by the second panel. Then the short wavelength fluctuations are generated, and eventually the field distribution stabilizes near the minimum of the effective potential, which corresponds to $\phi \sim 1$ in this figure. 
If one would use perturbation theory to estimate the total time which it takes for the decay of the homogeneous component of the scalar field following [1], one would find that this decay occurs only after $O\left(\lambda^{-1}\right) \sim 10^{12}$ oscillations for $\lambda \sim 10^{-12}$. It is quite amazing therefore that the nonperturbative effects lead to this decay within only 5 oscillations. The resulting field distribution after 5 oscillations, shown in the last panel of Fig. 6, is quite different from what one could expect on the basis of the perturbative approach to reheating or on the theory of parametric resonance in models of chaotic inflation.

As we see from Fig. 3, the occupation numbers of $\phi$-particles produced by the decaying inflaton field are exponentially large. This means that the initially homogeneous field $\phi$ decays into semi-classical waves of the field $\phi$. Since the main contribution to the occupation numbers of produced particles is given by particles (waves) with very small momenta, one could be tempted to conclude that the main reason of the rapid decay of the homogeneous component of the scalar field is the tachyonic preheating. On the other hand, the phase volume of these modes is relatively small. We have verified that for $v \sim 10^{-3}$ the main reason for the rapid decay of the homogeneous field $\phi$ in our computer simulations is the production of the particles with momenta $k \sim 0.5$ due to parametric resonance combined with the tachyonic effects, see Section III We expect, however, that for $v \ll 10^{-3}$, the main mechanism of the decay of the homogeneous mode will be related to the tachyonic preheating described in Section 【.

\section{THE FINAL STAGE OF REHEATING: DECAY OF THE INFLATON TO OTHER FIELDS}

So far we have described analytically and numerically how tachyonic preheating and parametric resonance cause the homogeneous inflaton field to rapidly decay into its own fluctuations. This process leads to the decoherence of the inflaton field and to spontaneous symmetry breaking, i.e. to the disappearance of the amplitude of oscillations of its homogeneous component. However, this is only the first part of reheating, which involves the decay of the inflaton into other degrees of freedom. In this section we discuss what happens when the inflaton is coupled to an additional field. We focus on the simplest case, a $g^{2} \phi^{2} \chi^{2}$ interaction with a second scalar field. Our basic conclusions, however, should be valid for 
a range of possible models including ones where reheating produces vectors and spinors as well as scalars.

Adding this field to the model gives us the potential

$$
V(\phi)=\frac{1}{4} \lambda \phi^{4}\left[\ln \frac{|\phi|}{v}-\frac{1}{4}\right]+\frac{1}{2} g^{2} \phi^{2} \chi^{2}+\frac{1}{16} \lambda v^{4} .
$$

It is known that when fluctuations of one field, here $\phi$, are excited to exponentially large occupation numbers, that will lead to rapid production of particles of other fields to which it is coupled [12]. In this case, however, this is complicated by the fact that the symmetry breaking vev of the inflaton field also produces an effective mass for the additional field $\chi$, which tends to suppress its production. The mass of $\phi$ in the minimum of its potential is given by $m_{\phi}=\sqrt{\lambda} v$, while the mass of $\chi$ is given by $m_{\chi}=g \phi=g v$. The parametric resonance near the minimum of $V(\phi)$ is rather narrow; it occurs only when $m_{\phi}>2 m_{\chi}$. Thus the condition for $\chi$ to be efficiently excited is $g^{2} \lesssim \lambda$.

But this means that the decay to particles $\chi$ occurs only if the decay rate is strongly suppressed by the small coupling constant $g^{2} \lesssim \lambda \sim 10^{-12}$.

Figure [7 shows the number density of particles of the $\phi$ and $\chi$ fields for this model. In both cases we take $v=10^{-3} M_{p}$, which we know produces efficient tachyonic preheating. In the case where $g^{2}=\lambda$ we see very little production of $\chi$ particles. When $g^{2}=\lambda / 10$, the $\chi$ field is driven up to exponentially large occupation numbers some time after the field $\phi$ grows. We also tested $g^{2}=100 \lambda$ and as expected found virtually no growth of the $\chi$ field.
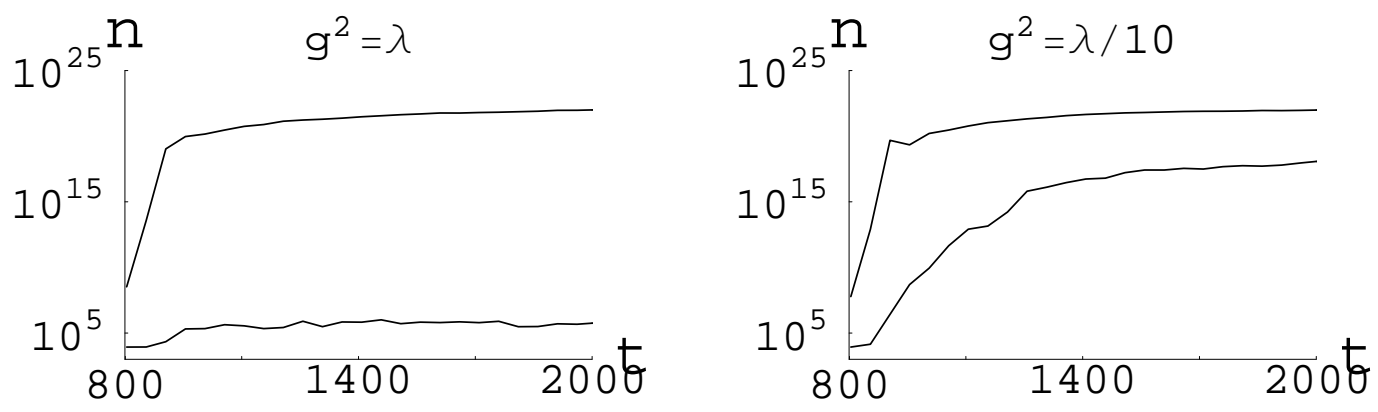

FIG. 7: Growth of the occupation numbers of $\phi$ and $\chi$ in the model (16). In both plots the upper curve shows $n_{\phi}$ and the lower curve shows $n_{\chi}$.

The results of our calculations show that even in the cases when the nonperturbative 
effects lead to the exponentially growing number of particles $\chi$, this number always remains exponentially smaller than the number of particles $\phi$. This means that nonperturbative effects in this simple model lead to a rapid decay of the nearly homogeneous oscillating field into particles or waves of this field, but not to the final stage of reheating when all particles of the field $\phi$ decay to other particles.

This suggests that the decay of the field $\phi$ eventually ends up by a perturbative stage when the field $\phi$ no longer oscillates coherently, and therefore one can use an approximation where each particle of the field $\phi$ decays independently, according to elementary theory of reheating developed in [1, 2]. In this case the final temperature of reheating is given by $T_{r} \sim \sqrt{\Gamma}$, where $\Gamma=\frac{g^{4} v}{8 \pi \sqrt{\lambda}}=\frac{g^{4} m}{8 \pi \lambda}$ is the rate of decay $\phi \rightarrow \chi \chi$ in our model [2]; $m=\sqrt{\lambda} v$.

Simple estimates based on the decay rates for this model calculated in 2] show that for $g^{2}=O(\lambda)$ the perturbative decay requires $O\left(\lambda^{-1}\right)$ oscillations, i.e. about $10^{12}$ oscillations in the model of new inflation with $\lambda \sim 10^{-12}$. The resulting temperature of reheating for $v \sim 10^{-3}, g^{2} \lesssim \lambda$ is $T_{r} \lesssim 10^{7} \mathrm{GeV}$.

Whereas this scenario of the last stage of reheating seems rather general, there could be some important exceptions. For example, some fields interacting with the inflaton field may remain light even though their interaction with the inflaton field is very strong. This is possible if their mass is protected by some symmetry or if their mass is small in the minimum of the effective potential due to some cancellation mechanism. In this case the decay rate of the inflaton field into such fields can be quite large, the final stage of reheating may also be very rapid, and the resulting reheating temperature can be much higher.

\section{CONCLUSIONS}

The first papers on reheating of the universe after inflation were devoted to investigation of this process in the new inflation scenario [1]. It was assumed that after inflation the nearly homogeneous scalar field oscillates for a very long time and eventually decays into particles of other fields in a process which can be described by a simple particle-by-particle decay of the scalar field $\phi$.

The subsequent investigation of reheating in chaotic inflation and in hybrid inflation have shown that reheating may occur much faster, due to nonperturbative effects such as 
parametric resonance [2] and exponential growth of tachyonic modes [3]. In this paper we study nonperturbative effects during reheating in new inflation.

We have found that for the simplest models of new inflation with a large amplitude of symmetry breaking, $v>10^{-2}$, the nonperturbative effects are relatively insignificant. After the first oscillation, the field $\phi$ never acquires the tachyonic mass. There can be a short stage of narrow parametric resonance, but it is inefficient, it shuts down very quickly, and the system enters the stage of perturbative particle-by-particle decay. A description of this regime can be found in Section IV of Ref. 2].

On the other hand, in the versions of new inflation with $v \lesssim 10^{-2}$, which includes the original new inflation model [4], the nonperturbative effects can be very powerful. As we have shown, a combination of tachyonic preheating and parametric resonance leads to a rapid dumping of energy of the oscillating inflaton field. For example, for $v \sim 10^{-3}$ the homogeneous oscillating inflaton field $\phi$ completely decays within 5 or 6 oscillations. For smaller values of $v$, the decay occurs even much faster.

However, during this decay, the homogeneous oscillating inflaton field $\phi$ decays to decoherent waves of the field $\phi$; its decay to the matter particles $\chi$ at this stage typically is rather inefficient. Therefore the rapid nonperturbative stage of preheating is not the end of the story but only a prelude to a lengthy stage of perturbative decay, which eventually gives the reheating temperature that could be calculated by the methods developed more than 20 years ago [1].

In this respect, the new inflationary scenario differs strongly from the simplest versions of chaotic inflation where the effect of parametric resonance and the subsequent violent stage of rescattering of produced particles makes thermalization much faster [12]. It differs even more from the hybrid inflation, where the process of decay of the zero mode of the inflaton takes just a single oscillation, and the subsequent stage of perturbative decay can occur relatively fast because the corresponding coupling constants there can be relatively large.

The main reason of the slow decay of the inflaton field is related to the fact that the fields with which the inflaton field $\phi$ interacts strongly are getting heavy because of this interaction (this does not happen in the simplest versions of the chaotic inflation scenario). As a result, the inflaton field can only decay to the particles with which it almost does not interact [13]. This strongly suppresses the decay probability and the resulting reheating 
temperature in the simplest models of new inflation. However, even in such models the reheating temperature can be sufficiently high for the subsequent stage of the low-scale baryogenesis.

\section{APPENDIX: PARAMETERS OF THE LATTICE CALCULATIONS}

The lattice simulation results shown in this paper were all calculated using LATTICEEASY [9], a publicly available program for simulating interacting scalar fields in an expanding universe. The model (11) is encoded in the "colemanweinberg.h" model file on the LATTICEEASY website [14]. The website contains documentation on using LATTICEEASY and details on the algorithms employed. In this appendix we simply report the parameters used for these runs. These parameters, along with the Coleman-Weinberg model file, should be sufficient for anyone to reproduce all of the results reported here.

The lattice spacing had to be chosen to be small enough to include modes with wavelengths $k>\sqrt{\lambda} v$, the effective mass at the minimum of the potential, while the total size of the box had to be large enough to include significant numbers of modes with $k<\sqrt{\lambda \ln \left(v / M_{p}\right)} v^{2} / M_{p}$, the tachyonic mass at the end of inflation. This moment, $\phi=v^{2} / M_{p}$, was taken as the start of the simulations. The initial velocity $\dot{\phi}$ was set to zero. Note, however, that the program uses rescaled variables

$$
\begin{aligned}
\phi_{\text {program }} & =a^{3 / 2} \frac{\phi}{v} \\
t_{\text {program }} & =\sqrt{\lambda} v t,
\end{aligned}
$$

so that in program units the initial values of $\phi$ and $\dot{\phi}$ are set to $v$ and $v^{2} \sqrt{3 \pi / 8}$. (This latter result accounts for the derivative of the scale factor as well as $\phi$.)

Most of the results in Section IV are for a run with $v=10^{-3} M_{p}$. For this run we used a one-dimensional grid of 16, 384 gridpoints, a total box size $L$ of 1500 , and a time step of 0.01 . We ran the simulation to a time $t=1000$ but only began recording spectra at $t=800$, the point when the field first started growing significantly. Various two-dimensional simulations were used to crosscheck many of the results in this section. The particular one shown in figure 6 was done on a $2048 \times 2048$ lattice using a time step of 0.05 (other parameters as just listed above). Note that all of these values are in program units. For example, $L=1500$ in 
program units corresponds to a physical box size of $1500 /(\sqrt{\lambda} v) \approx 5 \times 10^{12} M_{p}^{-1}$.

The results in Section $\nabla$ used the same parameters as the $v=10^{-3} M_{p}$ run described above, but with the addition of an extra field coupled to $\phi$ with coupling $g^{2}=\lambda$ or $g^{2}=\lambda / 10$.

Figure 1 shows results from a run with $v=10^{-1} M_{p}$. For this run we used 512 gridpoints, a total box size $L=40$, a time step of 0.01 , and a final time $t=100$.

\section{ACKNOWLEDGMENTS}

We would like to thank Lev Kofman for valuable comments. The work by J.M.K. was supported by the Stanford Graduate Fellowship and the Sunburst Fund of the Swiss Federal Institutes of Technology (ETH Zurich and EPF Lausanne). The work by A.L. was supported by NSF grant PHY-0244728. The work by M.D. was supported by a Schultz Foundation Fellowship.

[1] A. D. Dolgov and A. D. Linde, "Baryon Asymmetry In Inflationary Universe," Phys. Lett. B 116, 329 (1982); L. F. Abbott, E. Farhi and M. B. Wise, "Particle Production In The New Inflationary Cosmology," Phys. Lett. B 117, 29 (1982).

[2] L. Kofman, A. D. Linde and A. A. Starobinsky, "Reheating after inflation," Phys. Rev. Lett. 73, 3195 (1994) arXiv:hep-th/9405187; L. Kofman, A. D. Linde and A. A. Starobinsky, "Towards the theory of reheating after inflation," Phys. Rev. D 56, 3258 (1997) arXiv:hep-ph/9704452.

[3] G. N. Felder, J. Garcia-Bellido, P. B. Greene, L. Kofman, A. D. Linde and I. Tkachev, "Dynamics of symmetry breaking and tachyonic preheating," Phys. Rev. Lett. 87, 011601 (2001) arXiv:hep-ph/0012142 ; G. N. Felder, L. Kofman and A. D. Linde, "Tachyonic instability and dynamics of spontaneous symmetry breaking," Phys. Rev. D 64, 123517 (2001) arXiv:hep-th/0106179.

[4] A. D. Linde, "A New Inflationary Universe Scenario: A Possible Solution Of The Horizon, Flatness, Homogeneity, Isotropy And Primordial Monopole Problems," Phys. Lett. B 108, 389 (1982); A. Albrecht and P. J. Steinhardt, "Cosmology For Grand Unified Theories With 
Radiatively Induced Symmetry Breaking," Phys. Rev. Lett. 48, 1220 (1982).

[5] A. D. Linde, "Chaotic Inflation," Phys. Lett. B 129, 177 (1983).

[6] A. D. Linde, "Axions in inflationary cosmology," Phys. Lett. B 259, 38 (1991); A. D. Linde, "Hybrid inflation," Phys. Rev. D 49, 748 (1994) astro-ph/9307002.

[7] G. N. Felder, L. Kofman and A. D. Linde, "Inflation and preheating in NO models," Phys. Rev. D 60, 103505 (1999) arXiv:hep-ph/9903350.

[8] S. Y. Khlebnikov and I. I. Tkachev, "Classical decay of inflaton," Phys. Rev. Lett. 77, 219 (1996) arXiv:hep-ph/9603378; S. Y. Khlebnikov and I. I. Tkachev, "Resonant decay of Bose condensates," Phys. Rev. Lett. 79, 1607 (1997) arXiv:hep-ph/9610477.

[9] G. N. Felder and I. Tkachev, "LATTICEEASY: A program for lattice simulations of scalar fields in an expanding universe," arXiv:hep-ph/0011159.

[10] S. R. Coleman and E. Weinberg, "Radiative Corrections As The Origin Of Spontaneous Symmetry Breaking," Phys. Rev. D 7, 1888 (1973).

[11] J. H. Traschen and R. H. Brandenberger, "Particle Production During Out-Of-Equilibrium Phase Transitions," Phys. Rev. D 42, 2491 (1990).

[12] G. N. Felder and L. Kofman, "The development of equilibrium after preheating," Phys. Rev. D 63, 103503 (2001) arXiv:hep-ph/0011160; R. Micha and I. I. Tkachev, "Turbulent thermalization," Phys. Rev. D 70, 043538 (2004) arXiv:hep-ph/0403101.

[13] One can call this the Groucho Marx effect. The famous American comedian once announced that he would not want to belong to a club that would have him as a member. Similarly, the inflaton field in our model would decay to other particles only if it interacts with them very weakly, in which case the decay rate is strongly suppressed.

[14] http://www.science.smith.edu/departments/Physics/fstaff/gfelder/latticeeasy 